\title{
NSC-derived extracellular matrix-modified GeIMA hydrogel fibrous scaffolds for spinal cord injury repair
}

\author{
Zheng Chen', Lin Wang ${ }^{1}$, Chichi Chen ${ }^{1}$, Jie Sun ${ }^{1}$, Junchao Luo ${ }^{1,2}$, Wenguo Cui $\mathbb{B}^{3}$, Can Zhu' ${ }^{1}$ Xiaozhong Zhou ${ }^{4}$, \\ Xingzhi Liu (iD ${ }^{1,5}$, Huilin Yang ${ }^{1}$ and Qin Shi (1)
}

\begin{abstract}
Cell-derived extracellular matrix (ECM) has been applied in spinal cord injury (SCl) regeneration because of its various biological functions. However, insufficient mechanical properties limit its wide application. Herein, we developed GeIMA/ECM hydrogel fibrous scaffolds (GeIMA/ECM scaffolds) that can recruit and enhance the differentiation of neural stem cells (NSCs) by electrospinning and decellularization techniques. Moreover, the GelMA/ECM scaffolds had good mechanical properties and reinforced cell adhesion and proliferation. Compared to GelMA hydrogel fibrous scaffolds (GelMA scaffolds), GelMA/ECM scaffolds promoted more NSCs toward neurons by markedly enhancing the expression of MAP-2 and Tuj-1 and decreasing GFAP expression. In addition, the GelMA/ECM scaffolds significantly reduced the proportion of M1-phenotype macrophages, which is favorable for $\mathrm{SCl}$ repair. In vivo, the GelMA/ECM scaffolds recruited NSCs at the injured site, promoted neuron regeneration, and reduced the formation of glial scars and the inflammatory response, which further led to a significant improvement in the functional recovery of SCl.

Therefore, this scaffold shows potential in regenerative medicine, mainly in $\mathrm{SCl}$.
\end{abstract}

\section{Introduction}

Spinal cord injury (SCI) is a severe traumatic disease of the central nervous system that is accompanied by neurologic impairment and deterioration of various abilities. The inflammatory microenvironment caused by SCI induces the death of neurons and glial cells, which further prevents neural stem cells (NSCs) from differentiating into neurons in the injured site ${ }^{1}$. Due to the complex pathological mechanisms and low regenerative capacity after SCI, there is still no effective therapy against SCI. Currently, there have been some attempts to develop

\footnotetext{
Correspondence: Xingzhi Liu (617905296@qq.com) or

Huilin Yang (suzhouspine@163.com) or Qin Shi (shiqin@suda.edu.cn)

'Department of Orthopedics, The First Affiliated Hospital of Soochow University, Medical College of Soochow University, Orthopedic Institute of Soochow University, 899 Pinghai Road, 215031 Suzhou, Jiangsu, China 2Department of Orthopaedics, The People's Hospital of Suzhou National HiTech District (SND), 95 Huashan Road, 215219 Suzhou, Jiangsu, China Full list of author information is available at the end of the article These authors contributed equally: Zheng Chen, Lin Wang, Chichi Chen.
}

targeted therapy for SCI. One of the potential treatments is tissue engineering ${ }^{2}$. Tissue engineering can replace or restore damaged tissues by combining materials, cells and biologically active molecules ${ }^{3}$. Although there are many kinds of materials used in tissue engineering, such as poly (L-lactic acid) (PLLA) and poly (lactic-co-glycolic acid) (PLGA), they still have some disadvantages, such as a slow degradation rate, acidic degradation products and induction of inflammation at the implant site ${ }^{4-6}$. However, these materials showed no bioactivity and had difficulty reinforcing cell adhesion and proliferation. Therefore, it is necessary to fabricate a kind of scaffold with favorable bioactivity and biocompatibility.

Methacrylate gelatin (GelMA) is synthesized by gelatin and methacrylic anhydride ${ }^{7}$. This material has characteristics similar to those of the natural extracellular matrix (ECM), such as cell attachment sites and proteolytic degradability $^{7}$. GelMA has been widely used in treating $\mathrm{SCI}^{8}$. For instance, researchers used a three-dimensional

\section{(c) The Author(s) 2022}

(c) (i) Open Access This article is licensed under a Creative Commons Attribution 4.0 International License, which permits use, sharing, adaptation, distribution and reproduction cc) in any medium or format, as long as you give appropriate credit to the original author(s) and the source, provide a link to the Creative Commons license, and indicate if changes were made. The images or other third party material in this article are included in the article's Creative Commons license, unless indicated otherwise in a credit line to the material. If material is not included in the article's Creative Commons license and your intended use is not permitted by statutory regulation or exceeds the permitted use, you will need to obtain permission directly from the copyright holder. To view a copy of this license, visit http://creativecommons.org/licenses/by/4.0/. 
(3D)-GelMA hydrogel loaded with NSCs and bone marrow stromal cells (BMSCs) for SCI repair ${ }^{9}$. However, the low cell viability in vivo could be influenced by the local microenvironment. Liu et al. loaded stromal cell-derived factor-1 $\alpha$ (SDF1 $\alpha)$ and paclitaxel in GelMA scaffolds that synergistically promote vascular remodeling and spinal axon regeneration ${ }^{10}$. However, it was difficult to evenly mix factors, which led to the burst release phenomenon. Our previous research found that biomimetic GelMA scaffolds can mimic the ECM of the spinal cord and reduce the formation of glial scars ${ }^{11}$, but they lack cytokine components and cannot further promote repair in SCI. Therefore, a GelMA hydrogel scaffold capable of bioactivities is crucial for SCI regeneration.

The ECM plays an essential role in establishing and maintaining an appropriate microenvironment for tissue regeneration. The ECM provides structural support and regulates cellular functionalities, such as cell survival, proliferation, morphogenesis, and differentiation ${ }^{12,13}$. In addition, ECM regulates the signal transduction activated by cytokines and growth factors and inhibits inflammation ${ }^{14}$. At present, ECM has been widely applied in tissue engineering, such as $\operatorname{skin}^{15}$, bone ${ }^{16}$, cartilage bone ${ }^{17}$, and so on. Studies have shown that MSC-derived ECM regulates the polarization of macrophages to reduce inflammation and accelerate tissue regeneration. The ECM of olfactory ensheathing cells (OECs) facilitates neurogenesis and oligodendrocyte formation in NSCs ${ }^{18}$. Schwann cell-derived ECM can enhance myelination and provide nutritional support for axon regeneration ${ }^{19}$. Hydrogels with ECM derived from gray matter astrocytes reduce the formation of glial scars and the population of activated macrophages/ microglia and increase the penetration of regenerated axon fibers at the lesion $\operatorname{area}^{20}$. However, limited studies have focused on ECM derived from NSCs because it is challenging to collect ECM due to the round and suspended morphology of NSCs. Studies have revealed that the ECM derived from NSCs can be regulated by culturing these cells on the surface of different substrates ${ }^{21}$. Therefore, obtaining ECM derived from NSCs is important for fabricating scaffolds for SCI regeneration.

Currently, studies have found that the defects caused by $\mathrm{SCI}$ are unfilled, and NSCs are restricted at the edge of the injury. Additionally, the ECM cannot be used as a scaffold without integrating into materials, which is considered one of the limitations of ECM derived from cells in vitro. In this study, GelMA/ECM scaffolds were fabricated by electrospinning, UV curing, and decellularization technologies. These hydrogel scaffolds provided a favorable microenvironment for the differentiation and proliferation of NSCs and promoted SCI repair. First, the directional GelMA/ECM scaffolds facilitated the adhesion of NSCs and ECM deposition. Second, the GelMA/ECM scaffolds retained many tissue-specific growth factors and benefited the adhesion, proliferation, and differentiation of NSCs. Finally, GelMA/ECM scaffolds reduced inflammation and scar formation and recruited NSCs that differentiated into neurons to enhance SCI regeneration.

\section{Materials and methods}

Rats were kept in the Animal Experiment Center of Soochow University. The disposal complied with the 'Guiding Opinions on the Treatment of Experimental Animals and was approved by the Ethics Committee of Soochow University.

\section{Cell culture}

NSCs were prepared from the cerebral cortex of rats as previously described with minor modifications ${ }^{22}$. Briefly, the cerebral cortex was cut into pieces and digested in Accutase (Promocell, Germany). Then, the reaction was stopped by adding cold phosphate buffered saline (PBS), filtered through a $40 \mu \mathrm{m}$ cell strainer (BD, USA), and centrifuged at $1000 \mathrm{rpm}$. The cell suspension was cultured in a tissue culture flask (Corning, USA) in serum-free DMEM/F12 medium (HyClone, USA) containing $20 \mathrm{ng} /$ mL bFGF (Peprotech, USA), 10 ng/mL EGF (Peprotech, USA), and 1\% B27 (Thermo Fisher Scientific, USA). All cells were incubated in a $\mathrm{CO}_{2}$ incubator $\left(5 \% \mathrm{CO}_{2}, 37^{\circ} \mathrm{C}\right)$. The cells were identified by Nestin immunofluorescence staining (ab254048, Abcam, USA).

\section{Fabrication of GeIMA scaffolds}

GelMA was synthesized as described previously ${ }^{23}$. After freezing, GelMA was stored at $-80^{\circ} \mathrm{C}$. Two grams of GelMA was dissolved in $10 \mathrm{~mL}$ of hexafluoroisopropanol (HFIP, Aladdin, China) to form GelMA solution. Then, the solution was electrospun. The electrospinning conditions were as follows: voltage $15 \mathrm{kV}$, distance $20 \mathrm{~cm}$, flow rate $100 \mu \mathrm{L} / \mathrm{min}$ (min).

After electrospinning, the hydrogel fibers were placed in $10 \mathrm{~mL}$ of glutaraldehyde (Aladdin, China). After curing under UV light, the GelMA scaffolds were fabricated.

\section{Fabrication of GeIMA/ECM scaffolds}

NSCs were inoculated on GelMA/ECM scaffolds at a density of $2 \times 10^{5}$ cells $/ \mathrm{mL}$. After 14 days in culture, the scaffolds were washed with PBS and then repeatedly frozen and thawed. Finally, the scaffolds were stored at $4{ }^{\circ} \mathrm{C}$ after washing with PBS. The scaffolds were named GelMA/ECM scaffolds.

The ECM was prepared in the same manner except that NSCs were cultured in tissue culture plates (TCPs).

\section{Parameters of GeIMA and GelMA/ECM scaffolds}

The GelMA and GelMA/ECM scaffolds were lyophilized, and the morphology of the scaffolds was scanned by scanning electron microscopy (SEM, FEI, Netherlands). 
The water contact angles (WCAs) of the GelMA scaffolds were tested by a contact angle analyzer (Kruss, Germany).

Degradation experiment: The weight of the GelMA scaffolds was recorded as $W_{0}$. Then, the scaffolds were statically placed into DMEM/F12 medium at $37^{\circ} \mathrm{C}$ and $60 \mathrm{rpm}$. At predetermined time points, the scaffolds were weighed as $W_{\mathrm{t}}$.

$$
\text { Degradation rate }=\frac{W_{\mathrm{t}}-W_{0}}{W_{0}} * 100 \%
$$

Mechanical properties: GelMA and GelMA/ECM scaffolds were prepared, tensile properties were tested with a mechanical testing machine (Hengyi, China), and the Young's modulus was calculated.

The morphology of GelMA and GelMA/ECM scaffolds before and after freeze-drying was imaged with a microscope (Carl Zeiss, Germany).

\section{Characterization of GeIMA/ECM scaffolds}

Before and after freeze-drying, GelMA/ECM scaffolds were washed with PBS, incubated with DAPI at $37^{\circ} \mathrm{C}$ for $20 \mathrm{~min}$, and photographed with a fluorescence microscope (Carl Zeiss, Germany). Partial scaffolds were added to the lysate, and the steps of the kit were followed for DNA extraction (Tiangen, China). The concentration of DNA was detected by a Nanodrop 2000 (Thermo Fisher Scientific, USA).

Before and after freeze-drying, GelMA/ECM scaffolds were fixed with $4 \%$ paraformaldehyde (PFA) (Solarbio, China) and then incubated with anti-laminin antibody (ab7463, Abcam, USA) or anti-collagenIantibody (ab260043, Abcam, USA) overnight at $4{ }^{\circ} \mathrm{C}$. After removal of excess antibodies, the samples were incubated with secondary antibodies (ab150079, ab150077, Abcam, USA) at $37^{\circ} \mathrm{C}$ for $2 \mathrm{~h}$. A fluorescence microscope was used to collect the images.

\section{Biocompatibility of GeIMA/ECM scaffolds}

NSCs $\left(2 \times 10^{5}\right.$ cells $\left./ \mathrm{mL}\right)$ were seeded on the TCP, GelMA, and GelMA/ECM scaffolds. After 7 days, the cells were fixed with $4 \%$ PFA for 15 min and then stained with phalloidin (Invitrogen, USA). Some of the cells were stained with live/dead cell solution (Thermo Fisher Scientific, USA) for $30 \mathrm{~min}$. Morphological images of the cells were taken with a fluorescence microscope.

A Cell Counting Kit-8 (CCK-8, Dojindo, Japan) was used to assess cell proliferation. In brief, NSCs were incubated on the TCP, GelMA, and GelMA/ECM scaffolds at a density of $2 \times 10^{4}$ cells $/ \mathrm{mL}$ per well. The cells cultured on the tissue culture plate with growth medium were used as a control. After 3 days of culture, the samples were washed with PBS, and $10 \mu \mathrm{L}$ of CCK-8 in $100 \mu \mathrm{L}$ of DMEM/F12 was added. After $2 \mathrm{~h}$, the optical density (OD) was measured at $450 \mathrm{~nm}$ with a microplate reader (Biotek, USA).

\section{Differentiation of NSCs in GeIMA/ECM scaffolds}

NSCs were seeded on the TCP, ECM, GelMA, and GelMA/ECM scaffolds at a concentration of $2 \times 10^{5}$ cells/ $\mathrm{mL}$. After 14 days, the medium was removed, and the cells were washed twice with PBS. Then, NSCs were incubated with primary antibodies against $\beta 3$-tubulin (Tuj-1) (ab78078, Abcam, USA) and glial fibrillary acidic protein (GFAP) (ab33922, Abcam, USA) overnight at $4{ }^{\circ} \mathrm{C}$. After washing, secondary antibodies (ab150113, ab150079, Abcam, USA) and DAPI (ab228549, Abcam, USA) were added for $2 \mathrm{~h}$. Finally, the pictures were collected with a fluorescence microscope.

NSCs $\left(2 \times 10^{5}\right.$ cells $\left./ \mathrm{mL}\right)$ were seeded on the TCP, ECM, GelMA, and GelMA/ECM scaffolds. Total RNA was extracted from cells cultured for 14 days with TRIzol reagent (Beyotime, China). One microgram of RNA was reverse transcribed, and real-time PCR (RT-PCR) was carried out with a PCR System (Bio-Rad, USA). $\beta$-actin was used to normalize the expression levels of genes. The primer sequences are listed in Table $\mathrm{S} 1$. The relative gene expression was calculated by the $2^{-\Delta \Delta \mathrm{Ct}}$ method.

\section{Anti-inflammatory effects in GeIMA/ECM scaffolds}

BV2 cells $\left(25 \times 10^{4}\right.$ cells $\left./ \mathrm{mL}\right)$ were cultured in medium containing $1 \%$ FBS and LPS $(20 \mathrm{ng} / \mathrm{mL})$. After $24 \mathrm{~h}$ of culture, BV2 cells were seeded on the GelMA or GelMA/ ECM scaffolds at a concentration of $5 \times 10^{5}$ cells $/ \mathrm{mL}$. After 3 days, the cells were stained with anti-CD11b (101206, BioLegend, USA) and anti-CD86 antibodies (4300726, eBioscience, USA). Then, a flow cytometer assay (FCA) was performed (Thermo Fisher Scientific, USA).

\section{$\mathrm{SCl}$ modeling}

Forty-five female Sprague-Dawley rats (200-250 g) were anesthetized with pentobarbital sodium, and a $4 \mathrm{~mm}$ midline incision was made over the T10-T11 spinal region. The completely transected spinal cord $(3 \mathrm{~mm})$ was removed. After the scaffolds were transplanted, the muscle and skin were sutured. Forty-five rats were randomly divided into the sham, control, GelMA and GelMA/ECM scaffold groups. The control group was submitted to the surgical procedure only. The GelMA and GelMA/ECM scaffold groups were submitted to the surgical procedure and the implantation of GelMA or GelMA/ECM scaffolds. The sham group was subjected to midline incision exposure.

\section{Behavioral assessments}

For evaluation of locomotor function, the Basso, Beattie, Bresnahan (BBB) scale was used ${ }^{24}$. The BBB score was evaluated by investigators who were blinded. The score ranges from 0 to 21 . The test was started on the first day after surgery and then once a week for 8 weeks. 


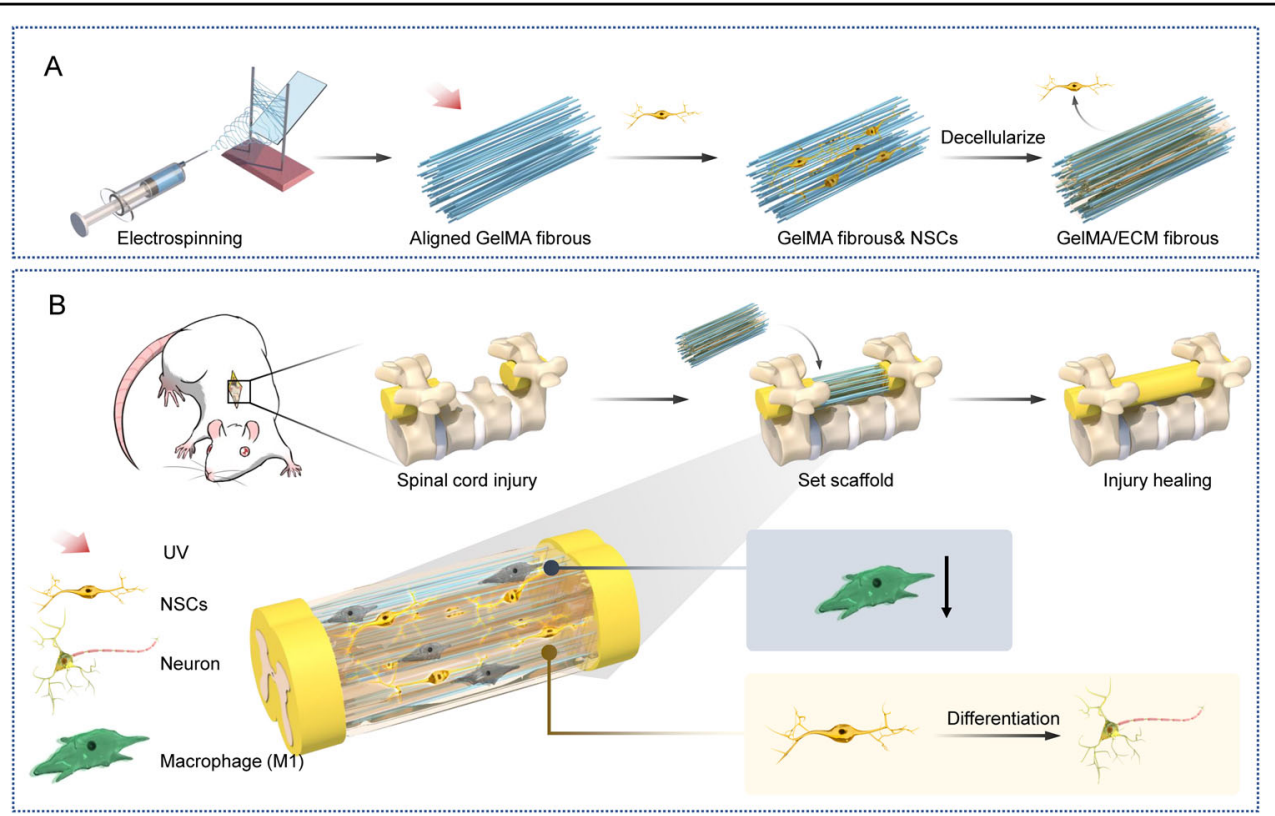

Scheme 1 Schematic illustration of NSC-derived extracellular matrix-modified GelMA scaffolds for spinal cord injury repair. GelMA/ECM scaffolds were developed by electrospinning, UV curing, and decellularization technologies. The GelMA/ECM scaffolds promoted NSCs toward neurons, decreased M1-phenotype macrophages and enhanced SCI repair.

\section{Histological analysis}

At 4 and 8 weeks, the samples were embedded in optimal cutting temperature compound (Sakura, USA) and cut into $14 \mu \mathrm{m}$ sections. After the samples were washed with PBS, they were blocked with goat serum and then incubated with primary antibody (NF-200, ab215903; Nestin, ab254048; Tuj-1, ab78078; GFAP, ab33922; CD68, ab955; CD206, ab64693; iNOS, ab178945; Abcam, USA) at $4{ }^{\circ} \mathrm{C}$ overnight. The next day, the samples were incubated with the secondary antibody (ab150079; ab150116; ab150077, Abcam, USA) and photographed.

\section{Statistical analysis}

All data were analyzed using SPSS statistical software (SPSS 22, IBM, USA). The values are represented as the mean \pm standard deviation (SD). All experiments were repeated three times unless otherwise indicated. A twotailed $t$ test was used for pairwise comparisons. One-way ANOVA followed by Tukey's post-test was used to evaluate the significance of the experimental data among more than three groups. A probability value $(p)<0.05$ was considered statistically significant.

\section{Results}

\section{Primary NSC isolation and identification}

To obtain ECM derived from NSCs, we first isolated neurons from the fetal rat brain cortex. Immunofluorescence indicated that the cells all expressed Nestin (Fig. S1) ${ }^{25}$.

\section{Characterization of the GeIMA/ECM scaffolds}

In this study, we prepared GelMA scaffolds by electrospinning and UV curing technology and then cultured NSCs on the surface of the GelMA scaffolds. With the RGD sequence, the NSCs adhered to the surface of hydrogels and secreted ECM. After 14 days of culture, the GelMA/ECM scaffolds were prepared by decellularization technology ${ }^{19}$ (Scheme 1).

To explore whether the freeze-drying process influences the morphology of scaffolds, we investigated the morphology and diameter of the GelMA scaffolds by SEM. The GelMA scaffolds were oriented with smooth surfaces (Fig. 1A). The average diameter of the fibers was $1.143 \pm$ $0.317 \mu \mathrm{m}$ before freeze-drying (Fig. 1C). After freezedrying, the fiber became thicker with a rougher surface (Fig. 1E). As shown by the WCA values, the GelMA surface was hydrophilic (Fig. 1B).

To prepare the GelMA/ECM scaffolds, we cocultured NSCs with the GelMA scaffolds for 14 days. Therefore, the degradation rate of the GelMA scaffolds was investigated. As shown in Fig. 1D, the GelMA scaffolds slowly degraded in the culture medium. As expected, the scaffolds still maintained $54.85 \pm 1.26 \%$ weight after 8 weeks of degradation. Herein, the GelMA scaffolds are suitable for subsequent ECM deposition.

The injured area of the spinal cord often suffers stretch, so the implantations should have appropriate mechanical properties. In this experiment, we tested the mechanical properties of the GelMA scaffolds before and after freezedrying. After freeze-thaw cycles, the GelMA scaffolds had 

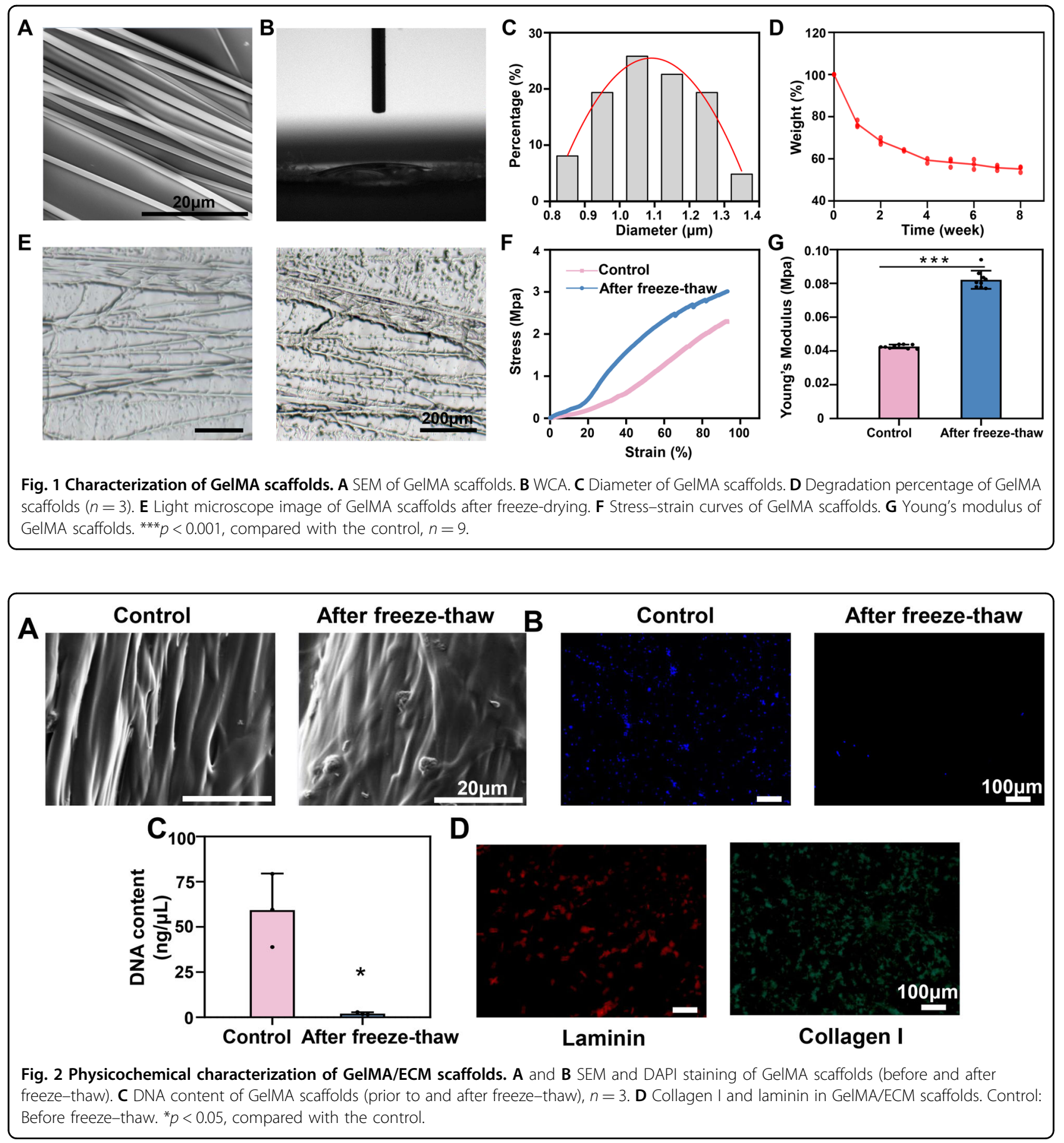

a higher stress level (Fig. 1F). The results showed that the Young's modulus was $0.04 \pm 0.001$ and $0.08 \pm 0.005 \mathrm{MPa}$ before and after the freeze-thaw cycles (Fig. 1G).

One of the disadvantages of decellularization technology is that the obtained ECM often contains cellular components that will cause immune responses ${ }^{26}$. Before freeze-drying, there were still some cells on the surface of the scaffold, but after freeze-drying, all the cells were eliminated (Fig. 2A). Similar results were also observed in the nuclear staining. Few cells were found on the scaffold after freeze-drying (Fig. 2B). Next, we evaluated the DNA content in the scaffold before and after decellularization. The concentrations of DNA before and after decellularization were $59.27 \pm 20.25$ and $2.03 \pm 0.76 \mathrm{ng} / \mu \mathrm{L}$, respectively, which indicated that decellularization technology can effectively remove the attached DNA of NSCs while retaining cellular components on the GelMA scaffolds ${ }^{19}$ (Fig. 2C). 


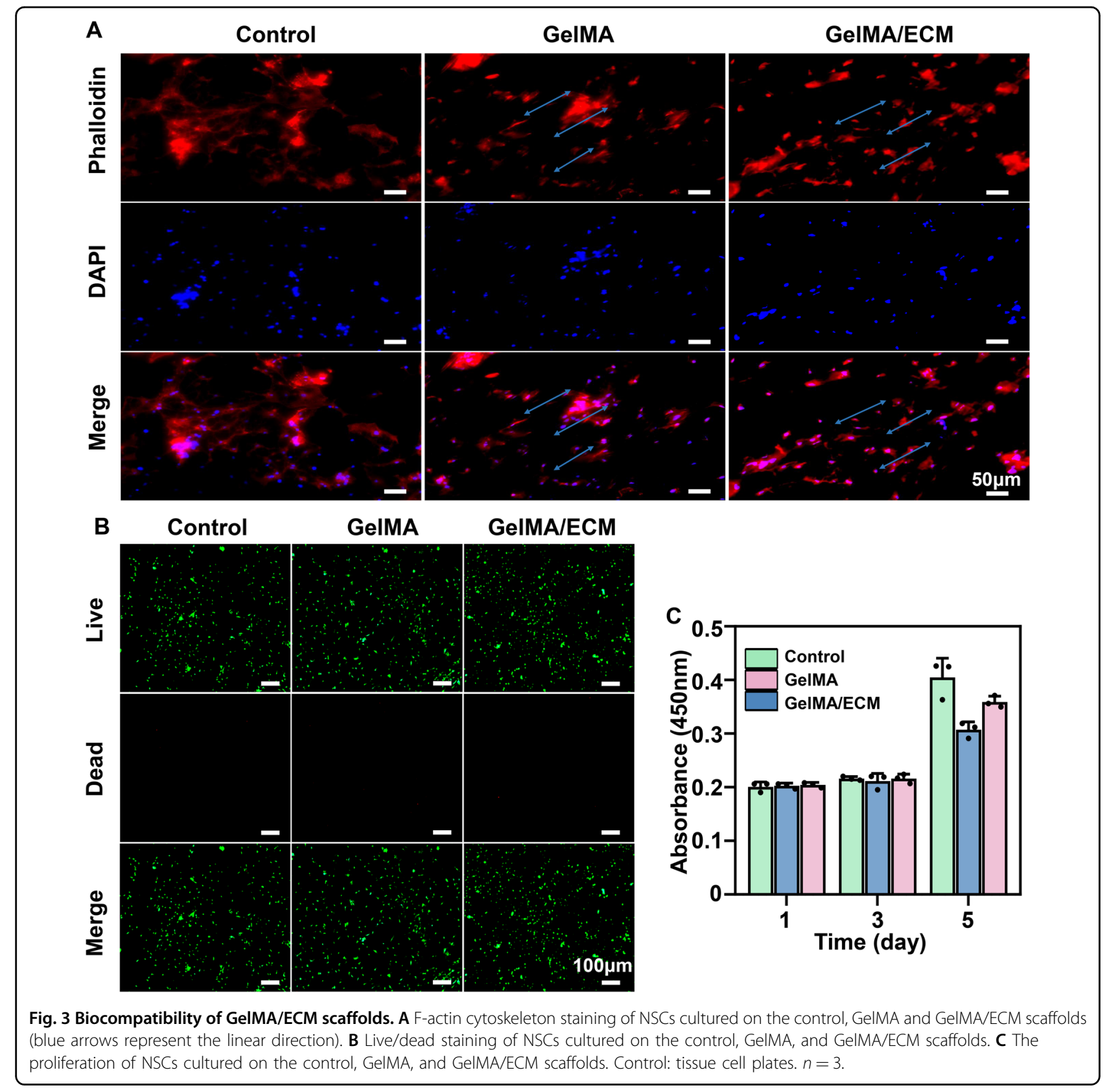

Collagen I and laminin are two main components of the $\mathrm{ECM}^{27}$. To verify the presence of ECM on the surface of the GelMA scaffolds, we performed immunofluorescence. As shown in Fig. 2D, the GelMA/ECM scaffolds exhibited biologically active ingredients, while no fluorescence signals were found on the GelMA scaffolds (data not shown).

\section{The GelMA/ECM scaffolds enhanced the adhesion and} differentiation of NSCs in vitro

The biocompatibility of the materials influences their application $^{28}$. After 7 days of coculture, the adhesion of NSCs on the surface of the GelMA/ECM scaffolds was evaluated by phalloidin staining.
Linear to the direction of the GelMA/ECM scaffolds, homogeneous distribution and favorable morphology of NSCs could be observed on the surface of the GelMA/ ECM scaffolds with its long axis parallel (Fig. 3A). The NSCs directionally proliferated according to the direction of hydrogel fibers. In contrast, NSCs were randomly distributed on the control group seeded on the TCP.

Live/dead staining revealed limited dead cells on the control, GelMA, and GelMA/ECM scaffolds (Fig. 3B). Then, CCK 8 assays were used to investigate the proliferation of NSCs on the GelMA/ECM scaffolds. The OD values were $0.204 \pm 0.005$ on Day $1,0.216 \pm 0.01$ on Day 3, and $0.359 \pm 0.011$ on Day 5 , indicating the favorable cell 


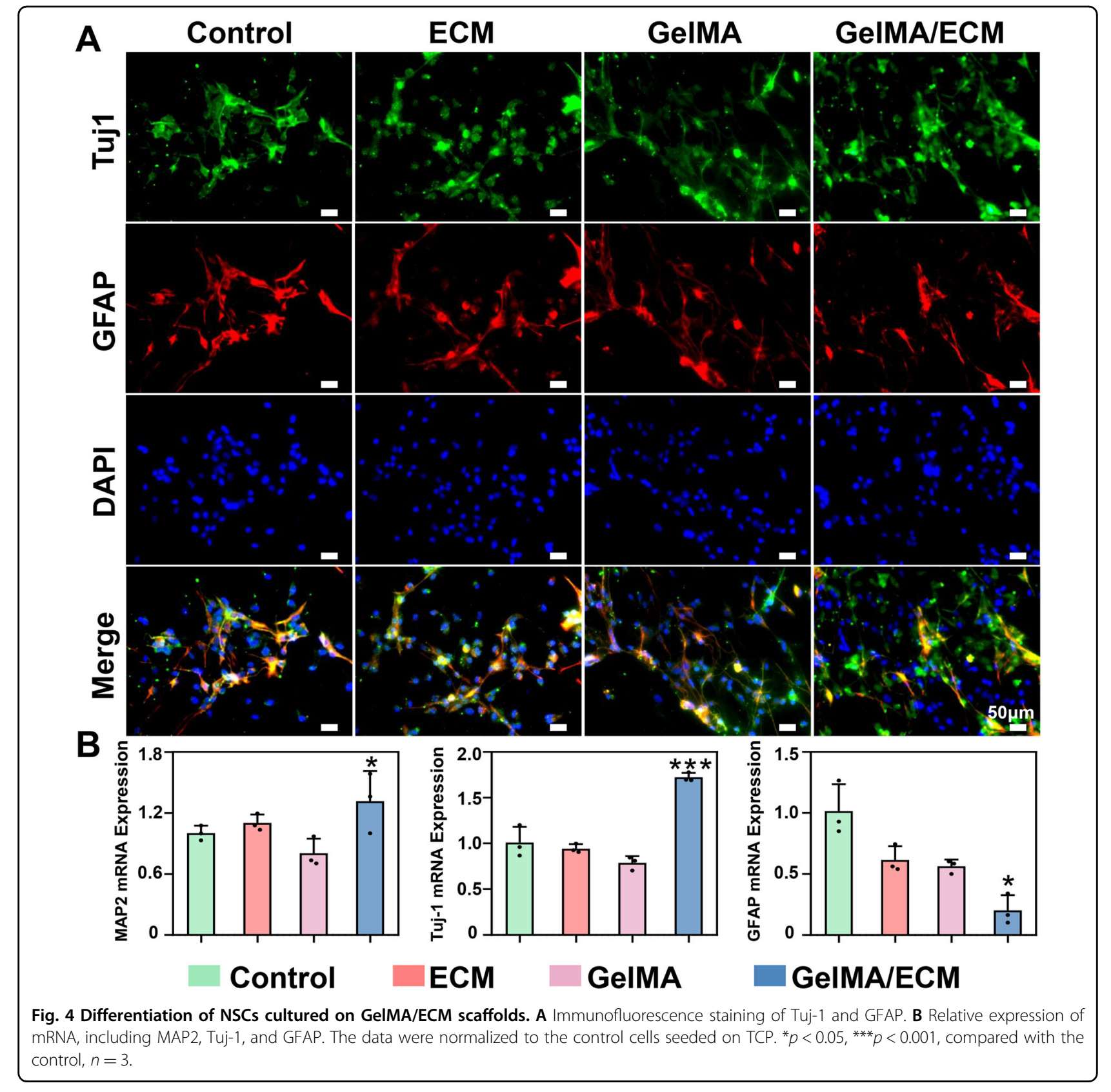

viability of NSCs cultured on the GelMA/ECM scaffolds (Fig. 3C). Interestingly, there was no difference in cell proliferation between the control and GelMA/ECM scaffolds. Therefore, it is speculated that the GelMA/ECM scaffolds can be transplanted into animals without affecting the proliferation of cells.

To analyze Tuj-1 and GFAP expression in NSCs, we performed immunofluorescence staining. After incubation, cells that were mostly positive for Tuj-1 with little positivity for GFAP were observed on the GelMA/ECM scaffolds (Fig. 4A).
In the next step, we applied RT-PCR to detect the expression of related genes on the NSCs cultured on the surface of GelMA/ECM scaffolds. The relative expression of microtubule-associated protein 2 (MAP-2) and Tuj-1 in the GelMA/ECM group was $1.32 \pm 0.3$ and $1.72 \pm 0.05$, respectively, which was remarkably higher than that in the control, ECM, and GelMA groups (Fig. 4B). Moreover, significantly reduced expression of the astrocyte marker GFAP was observed in the GelMA/ECM group $(0.20 \pm$ 0.12 ). These findings suggested that GelMA/ECM scaffolds promoted NSC differentiation into neurons. 


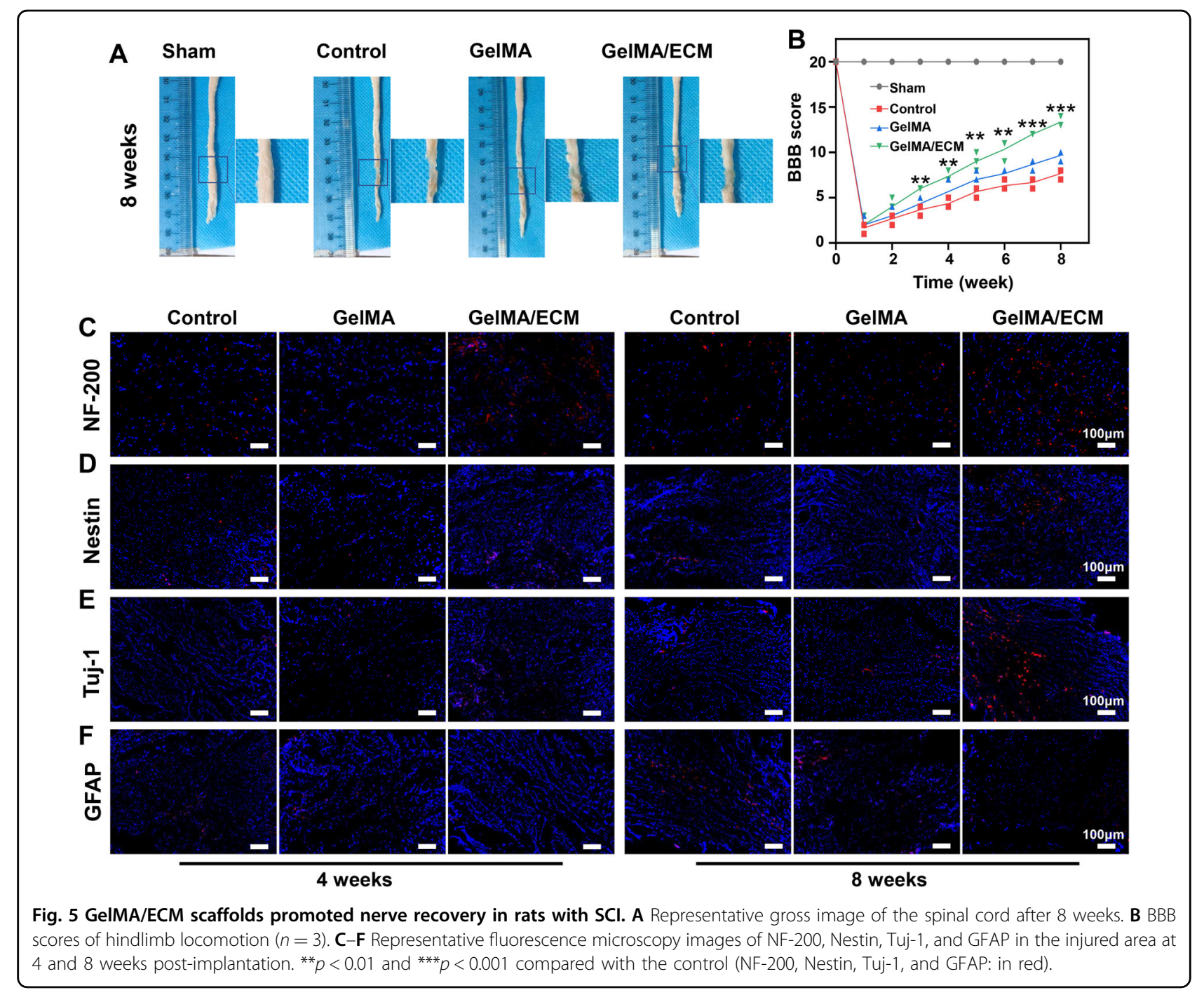

GelMA/ECM scaffolds reduced the proportion of M1phenotype BV2 cells in vitro

To evaluate the microglial polarization cultured on the scaffolds, we cultured BV2 cells on the TCP, ECM, GelMA, and GelMA/ECM scaffolds. The FCA results showed that the GelMA/ECM group expressed lower levels of CD86 than the other groups (Fig. S2).

\section{GeIMA/ECM scaffolds enhanced nerve function recovery in rats with $\mathrm{SCl}$}

The GelMA/ECM and GelMA scaffolds were implanted in the rats with SCI to explore the potential therapeutic effects. After 8 weeks, the T10-T12 spinal cords of the rats were collected. From the gross view, the spinal cords were intact in the sham group. In contrast, there were gaps at the jury sites of spinal cords in the control group. The GelMA and GelMA/ECM groups possessed a relatively intact spinal cord (Fig. 5A).
After the operation, BBB score analysis was performed to evaluate motor function recovery (Fig. 5B). Before the surgery, the motor function of all rats was healthy, with a BBB score of 21 points. After the surgery, the lower limbs were immediately paralyzed in all SCI groups, with a BBB score of 0 points. One week later, the motor function of these rats gradually recovered. The BBB score of the GelMA/ECM group increased slightly with time, but there was no significant difference among the three SCI groups. After 4 weeks, slowly increasing motor function scores were observed in the control group, and the recovery in the GelMA group was slightly better than that in the control group, but there was no significant difference between these two groups. Significantly increased scores were found in the GelMA/ECM groups. After 8 weeks, the recovery in the GelMA/ECM groups was dramatically better than that in the other two groups. 
The GeIMA/ECM scaffolds accelerate nerve regeneration in the rats with $\mathrm{SCl}$

NF-200 and Tuj-1 immunofluorescence staining was performed to evaluate the neurons in the injury area. The GelMA/ECM group had the most neurons in the damaged area, followed by the GelMA and control groups after 4 weeks. An increased number of neurons was observed in the GelMA/ECM group at 8 weeks compared with 4 weeks post-implantation. In addition, the GelMA/ ECM group exhibited the highest number of neurons relative to the other two groups (Fig. 5C, E).

Nestin is widely used as a specific marker in identifying NSCs. We found that the GelMA/ECM group had the largest proportion of Nestin-positive cells at 4 and 8 weeks post-implantation, indicating that the GelMA/ ECM scaffolds were beneficial for the migration of autologous NSCs to the injured site (Fig. 5D).

The formation of the glial scar is one of the main obstacles in promoting axon regeneration. To further investigate nerve repair, we used immunohistochemical staining to evaluate the expression of GFAP. The GFAPpositive cells in the control group were markedly higher than those in the GelMA and GelMA/ECM groups at 4 and 8 weeks post-implantation (Fig. 5F). The lowest number of GFAP-positive cells was observed in the GelMA/ECM group, indicating that the GelMA/ECM scaffolds reduced the deposition of scar components.

We performed CD68/CD206 and CD68/iNOS immunofluorescence staining to evaluate whether the GelMA/ ECM scaffolds significantly influence the immune microenvironment. As displayed in Fig. 6, the GelMA/ ECM group exhibited the highest ratio of CD206/CD68 and the lowest ratio of $\mathrm{CD} 68 / \mathrm{iNOS}$ at 4 weeks postimplantation. Eight weeks post-implantation, an increased ratio of CD68/CD206 was observed in each group, while the ratio of CD68/iNOS in the cells was decreased (Fig. 6).

In summary, these results indicated that implanting GelMA/ECM scaffolds reduced inflammation and provided a favorable microenvironment for NSC differentiation into neurons with good viability and functionality.

\section{Discussion}

In recent years, biomaterial scaffolds and ECM have been widely used to treat $\mathrm{SCI}$ in nerve tissue engineering ${ }^{29}$. The ECM is an ideal matrix for cell survival, proliferation, differentiation, and tissue remodeling. Generally, there are three main sources to obtain ECM. The first source of ECM is proteins, such as collagen, fibronectin, and laminin, with advantages such as pure and controllable components. The disadvantages of protein-derived ECM are also obvious. Proteins have difficulty mimicking the natural 3D structure of ECM. The second source is tissues or organs, which preserve the basic structure of the natural structure. Proteinderived ECM contains limited ECM protein components. In

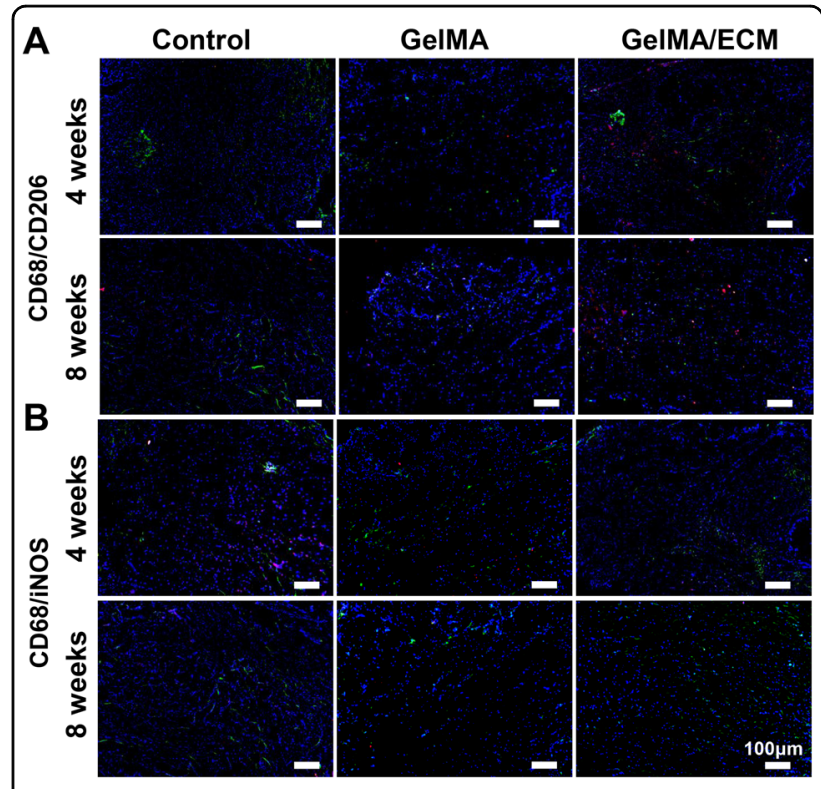

Fig. 6 GeIMA/ECM scaffolds reduced inflammation in vivo. A Representative fluorescence microscopy images of CD68/CD206 and CD68/iNOS. B At 4 or 8 weeks post-implantation (CD68: in green; CD206 and iNOS: in red).

contrast, the natural ECM in tissues consists of various structural proteins and cytokines. However, tissue-derived or organ-derived ECM still suffers some challenges, such as the weight of tissues, pathogens, and poor mechanical properties. The third source is the cell-derived ECM. Cellderived ECM has similar components to tissue-derived ECM without any pathogens. However, cell-derived ECM has limited mechanical strength. From this view, ECM is usually combined with biomaterials to obtain enhanced physical structure and mechanical properties.

In the present study, we used bionic GelMA hydrogel scaffolds to provide physical support for culturing NSCs. After 14 days of culture, the GelMA/ECM scaffolds were prepared by decellularization technology. The concentration of DNA is an important index for decellularization. After decellularization, the GelMA/ECM scaffolds exhibited a low DNA concentration of $2.03 \pm 0.76 \mathrm{ng} / \mu \mathrm{L}$, indicating successful decellularization and potentially limiting the host immune response.

GelMA is widely used in promoting SCI repair due to its excellent biocompatibility and mechanical properties ${ }^{8}$. Currently, the biocompatibility of GelMA has been proven by many studies in various animal disease models, such as bone defects, brain defects, and $\mathrm{SCI}^{8,10,23}$. NSCs cannot adhere to the TCP well in the standard culture process. However, they can stick to the surface of modified materials such as laminin ${ }^{30}$. GelMA is prepared by denatured collagen with an RGD motif ${ }^{31}$; thus, it promotes the adhesion of NSCs, which further proves the feasibility of GelMA/ECM scaffolds in SCI repair. In 
addition, NSCs proliferate well on the surface of GelMA/ECM scaffolds, suggesting that GelMA/ECM scaffolds can ensure cell proliferation when they are implanted into the host.

Promoting the neuronal differentiation of NSCs benefits the increased formation of neurons in SCI regeneration. Neurons establish neural connections between the implants and the host ${ }^{32}$. However, when autologous NSCs spontaneously migrate to the injury site, the microenvironment will cause cell apoptosis or make these NSCs differentiate into astrocytes rather than neurons ${ }^{33}$. Numerous active astrocytes release excessive chondroitin sulfate proteoglycan, tenascin, and intermediate filaments, leading to the formation of the glial scar, which further hinders axon regeneration $^{34}$. Therefore, the ideal biomaterial should guide the migration of endogenous NSCs by providing appropriate microenvironments and biosignals. At present, research focuses on transplanting NSCs to the injured site through predifferentiation. For example, Lai et al. prepared NT-3NSCs through transgenic technology, which was further combined with a scaffold to reinforce synaptic connections when these cells were transplanted into the host. The collagen scaffold carrying human placenta-derived mesenchymal stem cells (hPMSCs) facilitated the differentiation of autologous NSCs into neurons ${ }^{35}$. We found that the GelMA/ECM scaffolds promoted NSC differentiation into neurons. On the one hand, the directional hydrogel scaffolds filled the defect gap by simulating the internal structure of the spinal cord. On the other hand, ECM provided cytokines and a suitable microenvironment to accelerate autologous NSC migration and differentiation into neurons.

At the initial stage of SCI, the blood-spinal cord barrier is destroyed, and exogenous inflammatory cells (neutrophils, macrophages, monocytes, lymphocytes, and natural killer cells) are recruited, resulting in reactive oxygen species and increased inflammatory factor microglia ${ }^{36,37}$. Activated macrophages/microglia release various inflammatory factors, such as tumor necrosis factor- $\alpha$, interleukin-1 $\beta$, and interleukin- 6 , leading to inflammatory cascades. Additionally, these cytokines promote the apoptosis of neuronal cells, leading to serious secondary $\mathrm{SCI}^{38}$. Therefore, inhibiting the inflammatory response at the injury site contributes to the regeneration of SCI. In this study, we observed that the GelMA/ECM scaffolds could decrease M1-type BV2 cells. In vivo experimental results also proved that the GelMA/ECM scaffolds could reduce M1 macrophages/microglia. This finding is because the ECM on the GelMA/ECM scaffolds containing many factors can regulate the differentiation of macrophages, which can further protect the spinal cord from secondary injury. In addition, as a denatured product of collagen, GelMA has low antigenicity, which also helps to inhibit inflammation.

However, GelMA/ECM scaffolds still have some issues that could be improved for future clinical applications.
For example, GelMA modified with methacrylic anhydride may increase the complexity of the material. The batch-to-batch variability of ECM components affects SCI regeneration. Thus, the addition of supplemental biofactors may improve the therapeutic effect.

\section{Conclusion}

This research developed a new type of ECM scaffold that can recruit and enhance the differentiation of NSCs by using electrospinning, UV-curing, and decellularization technology. GelMA/ECM scaffolds mimic the spinal cord structure and mechanical properties, provide a suitable microenvironment to enhance NSC adhesion, promote NSC differentiation into neurons with recruited autologous NSCs, and reduce inflammation by an M2 phenotype shift, which further accelerates SCI regeneration in vivo. In general, our research provides a novel material-based strategy for SCI regeneration.

\section{Acknowledgements}

This work was supported by the National Natural Science Foundation of China (81972059, 81772358), the Key R \& D Programs of Jiangsu Province (BE2019668), and the Jiangsu Provincial Clinical Orthopedic Center, Priority Academic Program Development of Jiangsu Higher Education Institutions (PAPD). Strategic Priority Research Program of the Chinese Academy of Sciences (XDA16020104).

\section{Author details \\ 'Department of Orthopedics, The First Affiliated Hospital of Soochow University, Medical College of Soochow University, Orthopedic Institute of Soochow University, 899 Pinghai Road, 215031 Suzhou, Jiangsu, China. ${ }^{2}$ Department of Orthopaedics, The People's Hospital of Suzhou National Hi- Tech District (SND), 95 Huashan Road, 215219 Suzhou, Jiangsu, China. ${ }^{3}$ Shanghai Institute of Traumatology and Orthopaedics, Shanghai Key Laboratory for Prevention and Treatment of Bone and Joint Diseases, Ruijin Hospital, Shanghai Jiao Tong University School of Medicine, 197 Ruijin 2nd Road, 200025 Shanghai, China. ${ }^{4}$ Department of Orthopedics, The Second Affiliated Hospital of Soochow University, 1055 Sanxiang Road, 215003 Suzhou, Jiangsu, China. ${ }^{5}$ School of Nano-Tech and Nano-Bionics, University of Science and Technology of China, 96 Jinzhai Road, 230026 Hefei, Anhui, China}

\section{Author contributions}

C.Z., W.L., and C.C.C. performed the sample fabrication and measurement and wrote the manuscript. S.J., L.J.C., C.W.G., and Z.C. analyzed the data. Z.X.Z. and Y.H.L. supervised the project. L.X.Z. and S.Q. conceived the idea, designed the experiments and wrote the manuscript with review. All authors discussed the results and contributed to the paper.

Conflict of interest

The authors declare no competing interests.

\section{Publisher's note}

Springer Nature remains neutral with regard to jurisdictional claims in published maps and institutional affiliations.

Supplementary information The online version contains supplementary material available at https://doi.org/10.1038/s41427-022-00368-6.

Received: 27 October 2021 Revised: 26 January 2022 Accepted: 3 February 2022.

Published online: 4 March 2022 


\section{References}

1. Vroemen, M., Aigner, L., Winkler, J. \& Weidner, N. Adult neural progenitor cell grafts survive after acute spinal cord injury and integrate along axonal pathways. Eur. J. Neurosci. 18, 743-751 (2003).

2. Bedir, T., Ulag, S., Ustundag, C. B. \& Gunduz, O. 3D bioprinting applications in neural tissue engineering for spinal cord injury repair. Mater. Sci. Eng. C 110, 110741 (2020)

3. Li, G. et al. An NT-3-releasing bioscaffold supports the formation of TrkCmodified neural stem cell-derived neural network tissue with efficacy in repairing spinal cord injury. Bioact. Mater. 6, 3766-3781 (2021).

4. Walton, M. \& Cotton, N. J. Long-term in vivo degradation of poly-L-lactide (PLLA) in bone. J. Biomater. Appl. 21, 395-411 (2007).

5. Du, B. et al. Transplantation of artificial neural construct partly improved spinal tissue repair and functional recovery in rats with spinal cord transection. Brain Res. 1400, 87-98 (2011).

6. Ara, M., Watanabe, M. \& Imai, Y. Effect of blending calcium compounds on hydrolytic degradation of poly(DL-lactic acid-co-glycolic acid). Biomaterials 23, 2479-2483 (2002)

7. Zhao, Z. et al. Capturing magnesium ions via microfluidic hydrogel microspheres for promoting cancellous bone regeneration. ACS Nano $\mathbf{1 5}$ 13041-13054 (2021).

8. Fan, L. et al. Directing induced pluripotent stem cell derived neural stem cell fate with a three-dimensional biomimetic hydrogel for spinal cord injury repair. ACS Appl. Mater. Interfaces 10, 17742-17755 (2018).

9. Zhou, P. et al. Promoting $3 \mathrm{D}$ neuronal differentiation in hydrogel for spinal cord regeneration. Colloids Surf. B 194, 111214 (2020).

10. Zheng, Y. et al. Neuro-regenerative imidazole-functionalized GelMA hydroge loaded with hAMSC and SDF-1a promote stem cell differentiation and repair focal brain injury. Bioact. Mater. 6, 627-637 (2021).

11. Chen, $C$. et al. Bioinspired hydrogel electrospun fibers for spinal cord regeneration. Adv. Funct. Mater. 29, 1806899 (2019).

12. Paul, C. D. et al. Probing cellular response to topography in three dimensions Biomaterials 197, 101-118 (2019).

13. lijima, J., Konno, K. \& Itano, N. Inflammatory alterations of the extracellular matrix in the tumor microenvironment. Cancers (Basel) 3, 3189-3205 (2011).

14. Zou, D. et al. Preparation of a biomimetic ECM surface on cardiovascular biomaterials via a novel layer-by-layer decellularization for better biocompatibility. Mater. Sci. Eng. C 96, 509-521 (2019).

15. Zhou, Z. Q. et al. Adipose extracellular matrix promotes skin wound healing by inducing the differentiation of adipose-derived stem cells into fibroblasts. Int. J. Mol. Med. 43, 890-900 (2019).

16. Li, M. et al. Osteoblast/fibroblast coculture derived bioactive ECM with unique matrisome profile facilitates bone regeneration. Bioact. Mater. 5, 938-948 (2020).

17. Zhang, Y., Feng, G., Xu, G. \& Qi, Y. Microporous acellular extracellular matrix combined with adipose-derived stem cell sheets as a promising tissue patch promoting articular cartilage regeneration and interface integration. Cytotherapy 21, 856-869 (2019).

18. Cao, L. et al. Diffusible, membrane-bound, and extracellular matrix factors from olfactory ensheathing cells have different effects on the self-renewing and differentiating properties of neural stem cells. Brain Res. 1359, 56-66 (2010).

19. Gu, Y. et al. Chitosan/silk fibroin-based, Schwann cell-derived extracellular matrix-modified scaffolds for bridging rat sciatic nerve gaps. Biomaterials $\mathbf{3 5}$ 2253-2263 (2014)
20. Rose, J. C. et al. Biofunctionalized aligned microgels provide 3D cell guidance to mimic complex tissue matrices. Biomaterials 163, 128-141 (2018).

21. Sharma, K. D., Pandanaboina, S. C., Srivatsan, M. \& Xie, J. Y. Predominant differentiation of rat fetal neural stem cells into functional oligodendrocytes in vitro. Neurosci. Lett. 736, 135264 (2020).

22. Liu, G.I A novel limiting strain energy strength theory. Trans. Nonferrous Met. Soc. China 19, 1651-1662 (2009).

23. Qiao, Y. et al. Gelatin templated polypeptide co-cross-linked hydrogel for bone regeneration. Adv. Healthc. Mater. 9, e1901239 (2020).

24. Peng, Z. et al. Promotion of neurological recovery in rat spinal cord injury by mesenchymal stem cells loaded on nerve-guided collagen scaffold through increasing alternatively activated macrophage polarization. J. Tissue Eng. Regen. Med. 12, e1725-e1736 (2018).

25. Zhang, L. et al. NSCs migration promoted and drug delivered exosomescollagen scaffold via a bio-specific peptide for one-step spinal cord injury repair. Adv. Healthc. Mater. 10, e2001896 (2021).

26. Neves, S. C., Moroni, L., Barrias, C. C. \& Granja, P. L. Leveling up hydrogels: hybrid systems in tissue engineering. Trends Biotechnol. 38, 292-315 (2020).

27. Bosman, F. T. \& Stamenkovic, I. Functional structure and composition of the extracellular matrix. J. Pathol. 200, 423-428 (2003).

28. Yu, Q. et al. Chinese sesame stick-inspired nano-fibrous scaffolds for tumor therapy and skin tissue reconstruction. Biomaterials 194, 25-35 (2019).

29. Volpato, F. Z., Führmann, T., Migliaresi, C., Hutmacher, D. W. \& Dalton, P. D. Using extracellular matrix for regenerative medicine in the spinal cord. Biomaterials 34, 4945-4955 (2013).

30. Bruggeman, S. W. et al. Bmi1 controls tumor development in an Ink4a/Arfindependent manner in a mouse model for glioma. Cancer Cell 12, 328-341 (2007).

31. Antill-O'Brien, N., Bourke, J. \& O'Connell, C. D., Layer-by-layer: the case for 3D bioprinting neurons to create patient-specific epilepsy models. Materials (Basel) 12, 3218 (2019)

32. Lin, $\mathrm{X}$. et al. Cell transplantation and neuroengineering approach for spinal cord injury treatment: a summary of current laboratory findings and review of literature. Cell Transplant. 25, 1425-1438 (2016).

33. Li, X. et al. A collagen microchannel scaffold carnying paclitaxel-liposomes induces neuronal differentiation of neural stem cells through Wnt/ß-catenin signaling for spinal cord injury repair. Biomaterials 183, 114-127 (2018).

34. Yuan, Y. et al. Ethyl pyruvate promotes spinal cord repair by ameliorating the glial microenvironment. Br. J. Pharmacol. 166, 749-763 (2012).

35. Han, S. et al. Human placenta-derived mesenchymal stem cells loaded on linear ordered collagen scaffold improves functional recovery after completely transected spinal cord injury in canine. Sci. China Life Sci. 61, 2-13 (2018),

36. Tao, G. et al. Parthenolide promotes the repair of spinal cord injury by modulating M1/M2 polarization via the NF-KB and STAT 1/3 signaling pathway. Cell Death Discov. 6, 97 (2020).

37. Lima, R. et al. Systemic interleukin-4 administration after spinal cord injury modulates inflammation and promotes neuroprotection. Pharmaceuticals (Basel) 10, 83 (2017).

38. Wang, W. et al. Methane suppresses microglial activation related to oxidative inflammatory, and apoptotic injury during spinal cord injury in rats. Oxid. Med. Cell. Longev. 2017, 2190897 (2017). 